\title{
A Novel Biomaterial for Cartilage Repair Generated by Self-Assembly: Creation of a Self-Organized Articular Cartilage-Like Tissue
}

\author{
Kazuo Yudoh, Rie Karasawa \\ Institute of Medical Science, School of Medicine, St. Marianna University, Kawasaki, Japan. \\ Email: yudo@marianna-u.ac.jp
}

Received January $7^{\text {th }}, 2012$; revised February $24^{\text {th }}, 2012$; accepted March $11^{\text {th }}, 2012$

\begin{abstract}
Recently, attention has been drawn to tissue engineering and other novel techniques aimed at reconstruction of the joint. Regarding articular cartilage tissue engineering, three-dimensional materials created in vitro by cultivation of autologous chondrocytes or mesenchymal stem cells with a collagen gel have been implanted to replace defective parts of the articular cartilage in limited cases with the diseases such as trauma or arthritis. However, several passages of chondrocyte culture are required to obtain a sufficient number of cells for tissue engineering. Additionally, several other problems arise including dedifferentiation of chondrocytes during cell culture, which need to be solved from a viewpoint of cellular resources. The purpose of our study is to create a novel biomaterial possessing functions and structures comparable to native hyaline articular cartilage by utilizing the physicochemical properties of the cartilage matrix components themselves, in other words, employing a self-assembly technique instead of using chondrocytes to produce cartilage matrices eventually leading to articular cartilage tissue formation. We verified the conditions and accuracy of the selforganization process and analyzed the resulting micro structure using electron beam microscopy in order to study the technique involved in the self-organization which would be applicable to creation of cartilage-like tissue. We demonstrated that self-assembly of several cartilage components including type II collagen, proteoglycan and hyaluronic acid could construct self-assembled cartilage-like tissues characterized by nano composite structures comparable to human articular cartilage and by low friction coefficients as small as those of native cartilage.
\end{abstract}

Keywords: Self Assembly; Articular Cartilage; Tissue Engineering; Chondrocyte

\section{Introduction}

The joint destruction and symptom become aggravated with advance of disease as well as aging [1,2]. In the arthritis such as osteoarthritis (OA), tissue damage progresses with time after the onset and cartilage has less capacity for self repair, so that it is necessary to establish preventive measures as well as early treatment strategies against the degeneration of articular cartilage [3-5]. However, effective therapy for the cartilage degeneration still remains developing. It is therefore a matter of urgency to find new therapeutic agents and strategies that are clinically effective in treating age-associated musculoskeletal diseases in order to preserve high levels of activity of daily living in elderly people.

For the treatment of advanced joint destruction and degeneration, reconstruction of joints using new techniques such as tissue engineering are now attracting attention. Bone matrix-mimetic compounds that are pri- marily composed of hydroxyapatite have already been so well devised that they have sufficiently high affinity to the bone and enough rigidity similar to bone to be usable for the treatment of bone defects or bone destruction [6, 7]. Clinical application of bone in cases that suffer from large bone defects has already been realized and has been reported to yield good therapeutic results [7]. Since bone itself has superb ability of remodeling, grafted bone is claimed to be replaced by the host bone tissue through self-organization over a period of several weeks to months.

In contrast, materials suitable for cartilage repair remain to be created which have perfectly cartilage-specific tissue qualities represented by properties of elastic deformation and elastohydrodynamic lubrication. In recent studies, chondrocytes have been cultured in vitro in collagen or agarose gel to fabricate a three-dimensional material that can be implanted into a limited number of cases where subjects have a small posttraumatic cartilage 
defect of less than $3 \mathrm{~cm}^{3}$ in volume [8,9]. Some researchers are trying to obtain cell resources for cartilage tissue engineering by induction of differentiated chondrocytes from mesenchymal stem cells derived from the bone marrow [10].

Whether chondrocytes from patients' articular cartilage or inducing those cells from stem cells, it is essential for the formation of cartilage-like tissue that chondrocytes themselves should produce cartilage matrix. However, using current techniques, $2-5 \times 10^{6}$ chondrocytes are supposed to be required to fill a $1 \mathrm{~cm}^{3}$ cartilage deficit with cartilage-like tissue [8-10]. This requires continuous cell culture over several passages to obtain enough cell resources (number of cartilage cells). Many problems therefore remain to be solved from the viewpoint of cellular resources, since cultured cells may lose properties specific to the chondrocyte and may dedifferentiate during passages in culture. Moreover, it takes several weeks to engineer three-dimensional cultured tissue in a gel form using collagen gel. It then takes several months for this articular cartilage-like tissue to survive and become organized even when grafted in the defect site, since articular cartilage has a low capacity for repair [8]. Further studies are needed to verify whether the cartilage-like tissue manufactured by the existing techniques exhibit all the functions of cartilage such as low friction and load tolerance.

For the purpose of creating high performance articular cartilage-like tissue applicable to clinical use and overcoming the above weaknesses in cartilage repair, we focused on a novel biomaterial formed by self-assembly. Recently, new materials and devices have been developed through self-assembling various molecules at the nanometer scale by manipulating the intermolecular relations [11,12]. Molecules move randomly (Brownian motion) according to thermal motion and they may form a self-organized structure based on certain rules depending on the conditions of physicochemical properties such as inter-molecular avidity, surface modification, directions of covalent bonds, and ion arrangements. We considered it possible to create a novel biomaterial having functions as well as microstructures comparable to hyaline articular cartilage. We aimed to take advantage of the self-assembly technique in auto-organizing cartilage matrix components including collagen, proteoglycan and hyaluronic acid.

The purpose of this study is to develop a technique for creation of cartilage-like tissue not by cultivating chondrocytes to produce cartilage tissue via cartilage matrices, but by utilizing the physicochemical properties of the cartilage matrix components themselves, that is by selfassembly, without using cells.

\section{Materials and Methods}

\subsection{Self-Organized Cartilage-Like Material Formed from Hyaluronic Acid (HA), Proteoglycan (Aggrecan: AG), and Type II Collagen}

Solutions generated by mixing AG (Sigma-Aldrich, Inc., St. Louis, MO) at final concentrations of $0.1,0.25,0.33$, 0.5 or $1.0 \mathrm{mg} / \mathrm{ml}$ and HA (Chugai Pharmaseutical , Inc., Tokyo, Japan) at final concentrations of $1 \%, 2 \%, 3 \%, 4 \%$ or $5 \%$ were prepared with distilled-deionized water (DDW) at room temperature. Type II collagen (Collagen Research Center, Inc., Tokyo, Japan) was dissolved in DDW at final concentrations of $0.1,0.25,0.33$ or 0.5 $\mathrm{mg} / \mathrm{ml}$ at room temperature. The $\mathrm{pHs}$ of the $\mathrm{AG}+\mathrm{HA}$ solutions and the collagen solutions were adjusted to values from 4.0 to 11.0 in increments of 0.5 . Then, equal volumes of the $\mathrm{AG}+\mathrm{HA}$ and the collagen solutions were mixed at various combinations of $\mathrm{pH}$ and concentration and were incubated at $37^{\circ} \mathrm{C}$ in a humidified $95 \%$ air and $5 \% \mathrm{CO}_{2}$ atmosphere. After a variety of incubation periods followed by $30 \mathrm{~min}$ centrifugation at $20,000 \mathrm{rpm}$, aggregates formed in each mixture were subsequently scooped onto slide and observed using a digital high fidelity microscope VH-8000 (Keyence, Osaka, Japan). Also, aggregates were subjected to preparation as specimens for transmission electron microscopy.

\subsection{Formation of the Chondrocyte Conjugated Self-Organized Complex}

To exclude the influence of various factors in the serum that is usually added to culture medium on formation and maintenance of the complexes and on cell culture in them, nutridoma was added to serum-free medium (DMEM, Dako, Glostrup, Denmark) at a final concentration of 10\% nutridoma (Roche Diagnostics K.K., Tokyo, Japan) for cultivation of rat chondrocytes.

The self-organized AG/HA/collagen complex was washed three times with $10 \%$ nutridoma-DMEM, and then the complex was replaced with the same DMEM solution. Rat chondrocytes (Cellgarage, co. Ltd., Tokyo, Japan) were suspended in the above medium with the self-organized complex at a cell density of $5 \times 10^{4} / \mathrm{ml}$, followed by slight shaking and 3 minute centrifugation at $20,000 \mathrm{rpm}$. After centrifugation, the supernatant was removed, and the same DMEM solution was added. Then, the complex was incubated in new culture medium at $37^{\circ} \mathrm{C}$ in a humidified $95 \%$ air and $5 \% \mathrm{CO}_{2}$ atmosphere. Time course observation was carried out on the complex during incubation under a phase contrast microscope. After 6-week of incubation, some of the complexs were fixed for 2 days in $4 \%$ paraformaldehyde solution. Tissues were dehydrated in a series of ethanol solutions and 
infiltrated with xylene before being embedded in paraffin and cut into $6-\mu \mathrm{m}$ sections. Sections were deparaffinized through sequential immersion in xylene and a graded series of ethanol solutions in accordance with conventional procedures. The sections were stained with $\mathrm{Sa}-$ franin O-fast green or hematoxylin-eosin.

Other self-assembled complexes conjugated with chondrocytes were incubated at $37^{\circ} \mathrm{C}$ under $5 \% \mathrm{CO}_{2}$ for 24 weeks. The complexes were then subjected to specimen preparation for transmission electron and scanning electron microscopic study.

\subsection{Transplantation of the Self-Organized Complex into the Knee Joints of Experimental Animals}

The self-organized complex was prepared by the method described above. Eight 12-week-old male SD rats (Japan Charles-liver, Tokyo, Japan) were anesthetized and the right knee joints were aseptically dissected on both sides. Knee joint was shaved and disinfected with iodine solution (Isogene ${ }^{\circledR}$, Nippon Gene, Toyama, Japan). A medial parapatellar incision was made on the skin and a medial arthrotomy was performed. We prepared two sites of articular cartilage defects in each knee joint by punching the surface of the medial and lateral condyle with an 18 -gauge injection needle. The self-organized complex was then transplanted into those pits. The joint was washed with sterile saline and the joint capsule was closed. The contralateral side was also subjected to same surgical manipulation as a control.

After 24 weeks, we euthanized the animals to obtain the knee joints. Bone and cartilage tissue blocks were prepared for histological analysis. The paraffin-embedded tissue sections were stained with hematoxylin-eosin and Safranin-O. All experiments were conducted with the approval of the University Animal Care and Use Committee.

\section{Results and Discussion}

\subsection{A Self-Organized Cartilage-Like Complex Formed from HA, Proteoglycan (AG), and Type II Collagen}

We observed the aggregates in the AG + HA solutions at pHs ranging from 6 to 9 which were mixtures of AG at 0.25 to $0.5 \mathrm{mg} / \mathrm{ml}$ and $1 \%$ to $5 \%(\mathrm{v} / \mathrm{v}) \mathrm{HA}$. The aggregates were most clearly observed when the $\mathrm{pH}$ was in the range of 8 to 9 and when the concentration of AG was $0.33 \mathrm{mg} / \mathrm{ml}$ and HA was mixed in at 3\% (Figure 1(a)). The analysis with transmission electron microscopy revealed a fibrous structure and an aggregate structure where about 200 AG molecules were bound to an HA molecule (Figure 1(b)).

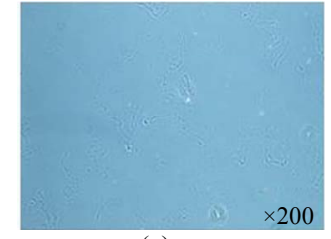

(a)

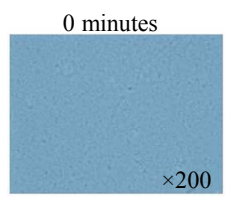

20 minutes

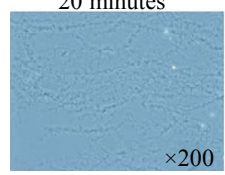

Collagen gel

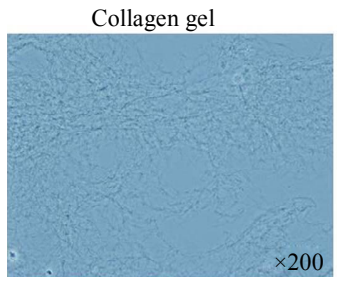

(d)

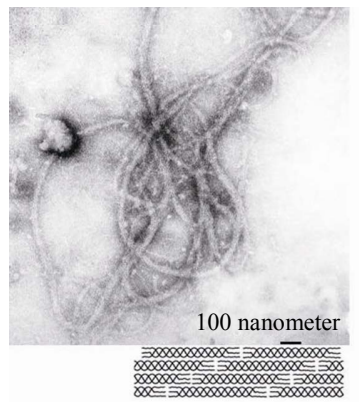

(e)

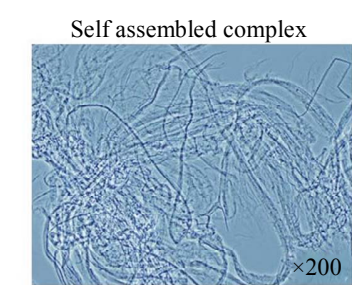

200 nanometer
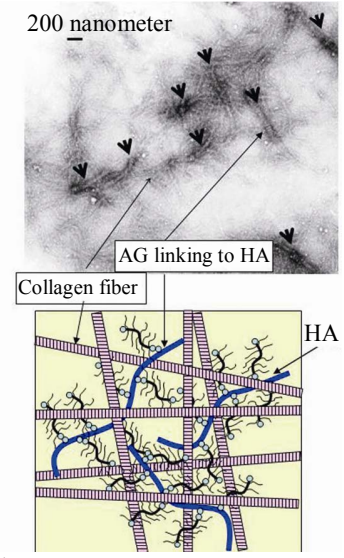

Figure 1. A self-assembled cartilage like complex from hyaluronic acid (HA), aggrecan (AG), and type II collagen. (a) The aggregates were formed in the AG + HA solutions at pHs ranging from 6 to 9 which erre mixtures of AG at 0.25 to $0.5 \mathrm{mg} / \mathrm{ml}$ and 1 to $5 \% \mathrm{HA}$; (b) The analysis with transmission electron microscopy showed an aggregate structure where about 200 AG molecules were bound to an HA molecule; (c) The self-assembled fibrous complex was formed in a time dependent manner; (d) The collagen gel was merely a gel form of collagen branches having no solid tissue structure. In contrast to the collagen gel, in the self-assembled complex, the collagen molecules were regu- larly bound to form long fibers; (e) The analysis by trans- mission electron microscopy showed that the self-organized collagen polysaccharide complex had a nano-composite structure in which the fibrous collagen was bound to AG linking to HA (arrow head), resembling cartilage tissue. 
Immediately after mixing the AG $+\mathrm{HA}$ solution with the collagen solution, collagen molecules started to gather around an AG + HA aggregate, followed by formation of collagen fibers bound inter-molecularly. The elongation and thickening of the fibrous collagen were observed over the time course of several minutes to several hours ( $2 \sim 3$ hours) (Figure 1(c)). The original collagen gel was merely a gel form of collagen branches having no solid tissue structure. In contrast to the collagen gel, the self-organized collagen polysaccharide complex has a nano-composite structure in which the fibrous collagen formed is bound to AG linking to HA, resembling cartilage tissue (Figure 1(d)).

We observed that the HA-AG/type II collagen selforganized complex consisted of $10 \sim 500 \mu \mathrm{m}$ long $(2 \sim$ $50 \mathrm{~nm}$ diameter) fibrous collagen at $\mathrm{pH}$ of 6 to 10 . Formation of this complex was most remarkable when the type II collagen molecule solution $(0.25 \sim 0.5 \mathrm{mg} / \mathrm{ml})$ was mixed in equal quantities with the $\mathrm{AG}(0.33 \mathrm{mg} / \mathrm{ml})$ + HA $(3 \%)$ solution. We confirmed by transmission electron microscopy that collagen molecules were regularly bound to form long fibers in the self-organized biomaterial (Figure 1(e)).

In previous studies on cartilage tissue engineering, numerous reports have indicated that differentiation, activation and proliferation of mature chondrocytes are, first of all, needed to induce the production of cartilage matrix components from the cells, resulting in tissue formation by self-assembly of matrix components. However, these procedures involve a long lead time before formation of articular cartilage tissue. Usually, it takes several weeks even to form a gel-like cartilage tissue. In contrast, using our self assembly technology the process is complete in just several hours. Our results indicate that the self-organized complex prepared in this short time might be a useful biomaterial for cartilage tissue engineering and for the treatment of articular cartilage defects.

\subsection{Observation of the Self-Organized Complex into Which Chondrocytes Were Transplanted}

The self-organized complex remained dense and that the chondrocytes were homogeneously present on the scaffold of fibers forming the complex. The chondrocytes were alive in the tissue on the basis of their extended dendrites on the surface of the complex. The self-organized complex at a high density and chondrocytes residing on or within the complex was observed in the scanning electron microscopic images. The chondrocytes were found to survive on the complex with dendrites extended (Figure 2(a)). The analysis by transmission electron microscopy revealed the intracellular tissue of inoculated chondrocytes within the inside the complex, suggesting survival of the chondrocytes in the tissue (Figure 2(b)).

In cartilage tissue engineering, an environment suitable for the long-term survival of chondrocytes is needed. In the present study, we demonstrated that our self-organized cartilage-like complex was able to perform functions of maintaining fluid components such as the most suitable culture medium for cell culture, which was characteristic of its three-dimensional net structure. The complex also provided a three-dimensional environment which served as a scaffold for chondrocyte growth.

\subsection{Transplantation of the Self-Assembled Cartilage Like Tissue into the Knee Joints of Experimental Animals}

We transplanted the self-assembled cartilage-like tissue into the rat OA model, partially defecting the articular cartilage tissue of the joint, and examined histologically the transplanted tissue over time (4 and 6 weeks after transplantation). The self-organized complex was found even at 6 months to maintain the same tissue structure as was initially observed. In cartilage tissue engineering, an environment suitable for the long-term survival of chondrocytes is needed (Figure 3).

There is much demand now for the establishment of a therapeutic system aimed at treating moderate to advanced disorders of joints, especially for cartilage repair in cases of bone and joint diseases such as OA for which no effective treatments are available at present other than arthroplasty (1-3). In the current study, we succeeded in manufacturing a material most suitable for cartilage tissue engineering by managing the self-organization mechanism on the nanometer scale. The biomaterial conjugated with chondrocytes was comparable to autologous cartilage in microstructure and consisted of hyaluronic acid, aggrecan and type II collagen.

\section{Conclusion}

Our self-organized cartilage-like tissue has a nano-com-

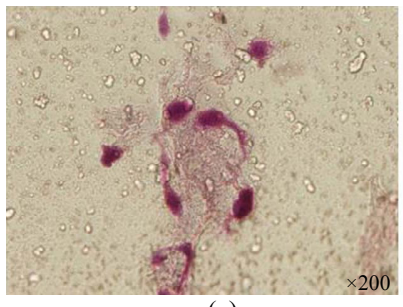

(a)

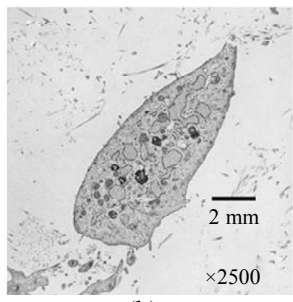

(b)
Figure 2. Observation of the self-assembled complex into which chondrocytes were transplanted. (a) The chondrocytes were present on the scaffold of fibers forming the complex; (b) The analysis by transmission electron microscopy showed the intracellular tissue of chondrocytes within the inside the complex. 

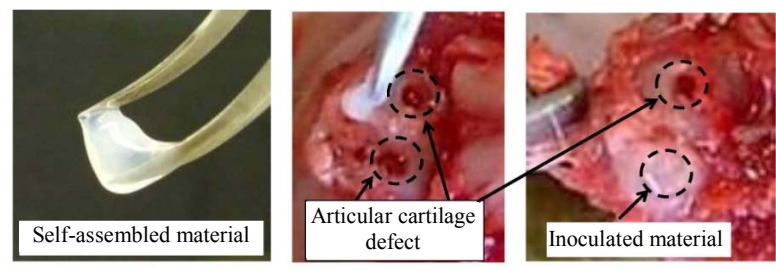

24 weeks after inoculation

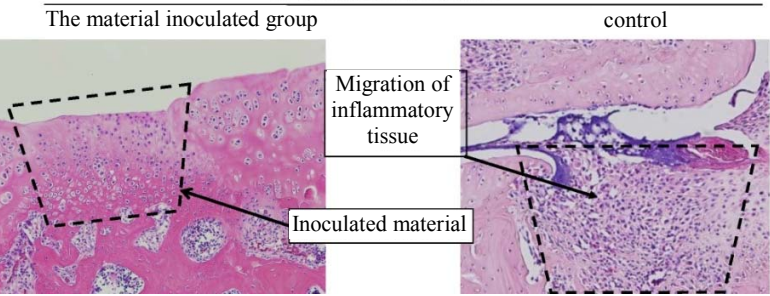

Figure 3. Representative images: transplantation of the selfassembled cartilage like tissue into the knee joints of experimental animals. Even after 24 weeks, the transplanted self assembled cartilage tissues were maintained in the joint and chondrocytes were observed in the inoculated material.

posite structure and is composed of components equivalent to native articular cartilage. It can, moreover, be manufactured in a short time of only a few hours. Our final goal is to develop a biomaterial most suitable for cartilage production. We emphasize the originality of this study, in which we do not need cellular resources like chondrocytes but only cartilage matrix components in order to produce cartilage-like tissue by the self-organization process.

\section{REFERENCES}

[1] J. A. Martin and J. A. Buckwalter, "Roles of Articular Cartilage Aging and Chondrocyte Senescence in the Pathogenesis of Osteoarthritis," Iowa Orthopaedic Journal, Vol. 21, 2001, pp. 1-7.

[2] R. F. Loeser, "Molecular Mechanisms of Cartilage Destruction: Mechanics, Inflammatory Mediators, and aging Collide," Arthritis \& Rheumatism, Vol. 54, No. 5, 2006, pp. 1357-1360. doi:10.1002/art.21813

[3] B. Kurz, A. K. Lemke, J. Fay, T. Pufe, A. J. Grodzinsky and M. Schunke, "Pathomechanisms of Cartilage Destruction by Mechanical Injury," Annals of AnatomyAnatomischer Anzeiger, Vol. 187, No. 5-6, 2005, pp. 473-

\section{5. doi:10.1016/j.aanat.2005.07.003}

[4] J. P. Pelletier, J. Martel-Pelletier and J. P. Raynauld, "Most Recent Developments in Strategies to Reduce the Progression of Structural Changes in Osteoarthritis: Today and Tomorrow," Arthritis Research \& Therapy, Vol. 8, 2006, p. 206. doi:10.1186/ar1932

[5] K. Yudoh, T. Nguyen, H. Nakamura, K. Hongo-Masuko, T. Kato and K. Nishioka, "Potential Involvement of Oxidative Stress in Cartilage Senescence and Development of Osteoarthritis: Oxidative Stress Induces Chondrocyte Telomere Instability and Downregulation of Chondrocyte Function," Arthritis Research \& Therapy, Vol. 7, 2005, pp. 380-391. doi:10.1186/ar1499

[6] I. O. Smith, L. R. McCabe and M. J. Baumann, "MC3T3E1 Osteoblast Attachment and Proliferation on Porous hydroxyapatite Scaffolds Fabricated with Nanophase Powder," International Journal of Nanomedicine, Vol. 1 No. 2, 2006, pp. 189-194. doi:10.2147/nano.2006.1.2.189

[7] P. F. Tang, G. Li, J. F. Wang, Q. J. Zheng and Y. Wang, "Development, Characterization, and Validation of Porous Carbonated Hydroxyapatite Bone Cement," Journal of Biomedical Materials Research Part B: Applied Biomaterials, Vol. 90, No. 2, 2009, pp. 886-893. doi: $10.1002 / \mathrm{jbm} \cdot$ b. 31360

[8] J. T. Kerker, A. J. Leo and N. A. Sgaglione, "Cartilage repair: Synthetics and Scaffolds: Basic Science, Surgical Techniques, and Clinical Outcomes," Sports Medicine \& Arthroscopy Review, Vol. 16, No. 4, 2008, pp. 208-216. doi:10.1097/JSA.0b013e31818cdbaa

[9] A. Getgood, R. Brooks, L. Fortier and N. Rushton, "Articular Cartilage Tissue Engineering: Today's Research, Tomorrow's Practice?" Journal of Bone \& Joint Surgery, (British Volume), Vol. 91, No. 5, 2009, pp. 565-567. doi:10.1302/0301-620X.91B5.21832

[10] C. J. Xian and B. K. Foster, "Repair of Injured Articular and Growth Plate Cartilage Using Mesenchymal Stem Cells and Chondrogenic Gene Therapy," Current Stem Cell Research \& Therapy, Vol. 1, No. 2, 2006, pp. 213229.doi:10.2174/157488806776956904

[11] G. Khushf, "Upstream Ethics in Nanomedicine: A Call for Research," Nanomedicine, Vol. 2, No. 4, 2007, pp. 511521. doi:10.2217/17435889.2.4.511

[12] M. Ebbesen and T. G. Jensen, "Nanomedicine: Techniques, Potentials, and ethical Implications," Journal of Biomedicine and Biotechnology, No. 5, 2006, Article ID: 51516. 